\title{
Bladder Rupture Following Surgical Mid-Trimester Abortion
}

\author{
Pallavee P, Samal S, Thulasi R, Ghose S \\ Department of Obstetrics \& Gynaecology, Mahatma Gandhi Medical College \& Research Institute, Puducherry,India
}

\section{DEAR EDITOR,}

A 25 years 2 nd gravida with previous normal vaginal delivery was admitted with complaints of excessive vaginal bleeding following surgical termination of pregnancy at 16 weeks of gestation at a private hospital. Clinical examination revealed mild abdominal distension and a bulky uterus with moderate amount of bleeding per vaginum.

Emergency ultrasound showed bulky uterus wth an empty cavity and free fluid in abdomen and pelvis. Abdominal X-Ray (erect view) didn't reveal any bowel perforation. Perurethral catheterisation showed frank hematuria. Patient was prepared for emergency laparotomy with a provisional diagnosis of uterine perforation with bladder injury.

Per operatively, hemoperitoneum of $1500 \mathrm{ml}$ and uterus enlarged to 14 weeks size with a vertical rent of $6 \mathrm{~cm}$ in the anterior wall. Bladder examination revealed $10 \mathrm{~cm}$ full thickness tear at the roof and $5 \mathrm{~cm}$ partial thickness tear in the anterior wall. (Fig. 1)

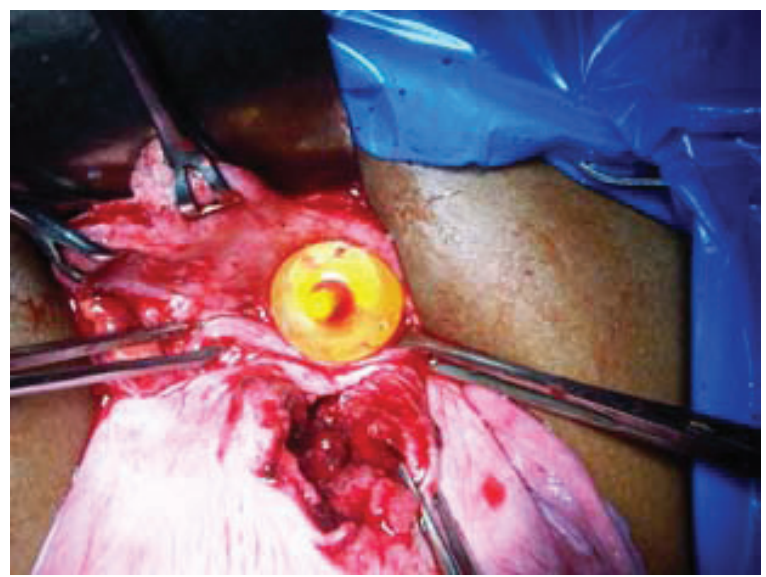

Fig 1. Rent in anterior wall of uterus and rupture of roof of urinary bladder with in-dwelling urinary catheter exposed.
Bowel exploration revealed no injury. Both the uterine and bladder rents were repaired. Suprapubic cystotomy and per urethral catheterization were done. Check cystogram on $14^{\text {th }}$ post-operative day revealed right vesico-ureteric reflux with no bladder leak. (Fig. 2)

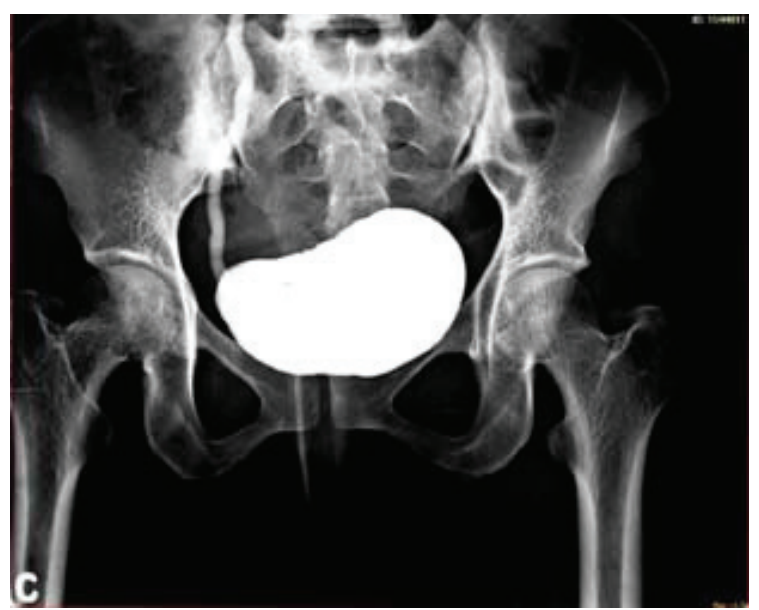

Fig. 2. Post-operative cystogram showing right vesicoureteric reflux, with no extravasation of dye from the bladder.

Bladder rupture following perforation of the uterus in association with elective mid-trimester abortion is an uncommon, but potentially serious complication. Such lacerations not only increase the immediate post-abortal morbidity but may also adversely influence future reproductive performance. Uterine perforation rates are highest in teaching settings and in cases of advanced parity and gestation. ${ }^{1}$

The exact incidence of such complication is not known. Early result of follow up study by Mackay et al showed increased risk of perforation of the myometrium with general anesthesia was reversed. ${ }^{2}$ In a large first trimester

\section{CORRESPONDENCE}

Dr. P. Pallavee

Department of Obstetrics and Gynaecology

Mahatma Gandhi Medical College \& Research Institute

Puducherry (India)

Phone: 9489693270

Email: ppallevee@rediffmail.com 
study performed by Hakim-Elahi et al $26 \%$ of patients underwent general anesthesia with intravenous bolus methohexital, uterine perforation occurred in only 0.09 per 1,000 cases. $^{3}$ However, perforation of the myometrium may be unrecognized and bladder rupture following such a complication may be underreported. Kaali et al ${ }^{4}$ found that only two of 14 uterine wall perforations were recognized in a series of 706 patients undergoing concurrent firsttrimester vacuum abortion and laparoscopic sterilization. Suspicion of perforation is raised when instruments pass farther than expected, often without discernible resistance; when bleeding is excessive, as in the case under discussion; or when contact with the gritty surface of the endometrium is lost during aspiration or curettage.

The likelihood of perforation is greater if the uterus is retroverted. The most frequent site of myometrial perforation with all types of intrauterine surgery is the relatively avascular anterior or posterior midline surface

\section{REFERENCES}

1. Grimes DA, Schulz KF, Cates W Jr. Prevention of uterine perforation during curettage abortion. JAMA. 1984;251(16):210811.

2. Mackay HT, Schulz KF, Grimes DA. Safety of local versus general anesthesia for second-trimester dilatation and evacuation abortion. Obstet Gynecol. 1985;66(5):661-5.

3. Hakim-Elahi E, Tovel HMM, Burnhill MS. Complications of firsttrimester abortion: A report of 170,000 cases. Obstet Gynecol. 1990;76(1):129-35. of the active segment. Perioperative ultrasound may be useful in documenting whether a perforation has occurred and in monitoring the cul-de-sac for accumulation of blood $^{5}$ or urine, if bladder rupture occurs as in our patient. Conservative protocols for patients with known or suspected low-risk perforations have been advocated by a variety of authors. ${ }^{4}$ However, if considerable intraabdominal damage has been caused by instruments, especially suction and sharp curettes, passed through a uterine defect into the peritoneal cavity, as in the present case, laparotomy to examine the abdominal contents is often the safest course of action.

4. Kaali SG, Szigetvari IA, Bartfai GS. The frequency and management of uterine perforations during first trimester abortions. Am J Obstet Gynecol. 1989;161(2):406-8.

5. Lajinian S, Margono F, Mroueh J. Sonographic appearance of suspected iatrogenic uterine perforation: a case report. J Reprod Med. 1994;39(11):911-2. 